\title{
DÉVALAISON DES SMOLTS ET EFFICACITÉ D'UN EXUTOIRE DE DÉVALAISON A L'USINE HYDROÉLECTRIQUE D'HALSOU SUR LA NIVE
}

\author{
M. LARINIER et Sylvie BOYER-BERNARD
}

Cellule Passes à Poissons CSP-CEMAGREF, BP 26, 31321 CASTANET-TOLOSAN Cedex

\section{RÉSUMÉ}

La dévalaison des smolts de saumon atlantique (Salmo salar) à la centrale hydroélectrique d'Halsou sur la Nive a été suivie en 1987 et 1988 afin de tester l'efficacité d'un exutoire de surface destiné à éviter le transit des poissons par les turbines. Les rythmes d'activité quotidienne, nycthémérale et horaire ont été relevés par piégeage et comptage vidéo. L'efficacité du clapet a été estimée par marquage et recapture : entre $42 \%$ et $95 \%$ des poissons marqués ont emprunté le clapet de dévalaison. L'influence du débit transitant par le clapet sur l'efficacité de celui-ci a été mise en évidence. Différents types d'éclairage (halogène, vapeur de mercure) ont été testés à titre exploratoire. Les observations visuelles montrent que les poissons sont attirés par la lumière à vapeur de mercure. Ils se maintiennent toutefois hors de la zone d'éclairement maximal : les passages à l'exutoire sont significativement plus importants lorsque celui-ci est laissé dans l'obscurité que lorsqu'il est directement éclairé par les lampes halogène ou fluorescentes à vapeur de mercure.

\section{DOWNSTREAM MIGRATION OF SMOLTS AND EFFECTIVENESS OF A FISH BYPASS STRUCTURE AT HALSOU HYDROELECTRIC POWERHOUSE ON THE NIVE RIVER}

\section{SUMMARY}

Downstream migration of Atlantic salmon (Salmo salar) smolts in river Nive SouthWest France was studied in 1987 and 1988 to access the effectiveness of a fish bypass structure at the hydroelectric plant of Halsou. Passage of fish was determined by trapping and video recording. Daily, diurnal and hourly passages at bypass were determined. Tests with marked fish showed between $42 \%$ and $95 \%$ of the smolts used the surface bypass. Significantly more smolts were bypassed when the discharge was increased. Exploratory tests with halogen and mercury lights were performed. Visual observation indicated that fish was attracted to the lights but avoided the point source : results showed an increased rate of passage when the lamps lighting up the bypass were turned off.

\section{INTRODUCTION}

Le problème de la dévalaison des juvéniles de saumons et de truites de mer se pose en France sur de nombreuses centrales hydroélectriques situées sur les cours d'eau à migrateurs. La conception de dispositifs de franchissement vers l'amont (passes à poissons) a fait l'objet d'une attention toute particulière depuis une douzaine d'années. II convenait d'aborder ensuite l'étude des dispositifs permettant aux juvéniles d'éviter le 
passage par les turbines, le but poursuivi à terme étant la définition d'un certain nombre de dispositifs-types et de leurs critères de dimensionnement. Parmi les dispositifs envisageables, celui qui paraît incontestablement le plus simple à mettre en œuvre, en particulier sur les ouvrages existants, est l'exutoire de surface convenablement placé par rapport aux grilles de protection de la centrale (EPRI,1986 ; RUGGLES et HUTT, 1984). Si de tels dispositifs ont déjà été installés sur plusieurs sites (FARWELL, 1972 ; DAVIS et FARWELL, 1975 ; CONRAD, 1986 ; NETTLES et GLOSS, 1987), ils n'ont cependant jamais fait à notre connaissance l'objet de tests systématiques et leur efficacité réelle n'a été que très rarement évaluée (SEMPLE et MC LEOD, 1976 ; SEMPLE, 1979 ; BOMASSI et TRAVADE, 1985).

La centrale hydroélectrique d'Halsou, située sur la Nive, semblait un site particulièrement favorable à l'expérimentation d'un tel dispositif, du fait de la présence d'une population de juvéniles de saumon suffisamment abondante (BOUSQUET, 1981, 1988) et de l'aménagement récent d'un clapet destiné à améliorer les conditions de dévalaison.

L'objet de l'étude (BERNARD et LARINIER, 1991) était d'essayer de rationaliser l'emploi d'un clapet de décharge comme exutoire de dévalaison en examinant en particulier l'influence du débit sur son efficacité, et de tester à titre exploratoire l'influence de plusieurs sources de lumières sur le comportement de dévalaison nocturne des smolts.

\section{MATÉRIEL ET MÉTHODES}

\subsection{Site d'expérimentation}

La centrale hydroélectrique d'Halsou (fig. 1) est située sur le cours inférieur de la Nive, affluent rive gauche de l'Adour. Un barrage poids de $172 \mathrm{~m}$ de largeur et de $2.50 \mathrm{~m}$ de hauteur (cote de la retenue normale 13.00 NGF) dérive les eaux de la Nive dans un canal d'amenée de $925 \mathrm{~m}$ de long et de $11 \mathrm{~m}$ de large. Le canal s'évase au niveau de la centrale équipée de 3 roues Francis horizontales doubles turbinant au maximum $30 \mathrm{~m}^{3} / \mathrm{s}$ sous $4.25 \mathrm{~m}$ de chute nette. La largeur du plan de grilles est d'une vingtaine de mètres.

Le régime des eaux de la Nive est de type pluvial. II se caractérise par de hautes eaux en hiver et au début du printemps et par des basses eaux de juillet à octobre. Le module interannuel est voisin de $36 \mathrm{~m}^{3} / \mathrm{s}$ à Halsou. Les débits mensuels moyens des mois de mars, avril et mai, mois correspondant à la période de dévalaison des smolts, sont respectivement de $48 \mathrm{~m}^{3} / \mathrm{s}, 53 \mathrm{~m}^{3} / \mathrm{s}$ et $42 \mathrm{~m}^{3} / \mathrm{s}$.

Le clapet de décharge utilisé pour la dévalaison est situé en rive droite à proximité des grilles de protection de la prise d'eau. Sa largeur est de $1.38 \mathrm{~m}$, sa hauteur de $0.90 \mathrm{~m}$. Il est situé à l'aval d'un court radier en béton d'une longueur variant, de la rive gauche à la rive droite, de $1.40 \mathrm{~m}$ à $0.80 \mathrm{~m}$. La cote moyenne de ce radier est $12.20 \mathrm{NGF}$, c'est-à-dire à $50-60 \mathrm{~cm}$ en-dessous des niveaux d'eau les plus fréquemment observés dans le canal d'amenée. La cote de déversement du clapet peut être réglée par l'intermédiaire d'un câble et d'une manivelle. Un bassin, à l'aval immédiat de la vanne, d'une profondeur de $1.20 \mathrm{~m}$, a été aménagé au printemps 1987 afin d'améliorer les conditions de réception des juvéniles après leur passage par le clapet (fig. 2). Un dispositif de piégeage situé à l'aval du bassin de réception permet de comptabiliser les smolts transitant par le clapet.

\subsection{Campagnes de suivi du clapet}

Bien qu'étant prévue à partir de la seconde quinzaine de mars, la campagne 1987 n'a pu débuter qu'à la mi-avril en raison des travaux relatifs à la construction du bassin de réception puis d'une crue importante qui interdisait tout accès au système de piégeage. Les piégeages n'ont pu être effectués que 31 jours et se sont déroulés du 16 avril au 16 mai sans interruption.

La campaane 1988, commencée le 9 mars, s'est achevée le 13 mai (ce qui représente 66 jours d'expérimentation) et a englobé l'ensemble de la période de dévalaison. Au cours de la campagne, le piégeage a dû être interrompu 14 jours, à quatre 
reprises, sur des périodes variant de 1 à 9 jours suite à une succession de crues pendant lesquelles le piège ne pouvait fonctionner. L'exutoire de dévalaison a donc été testé par piégeage pendant 52 jours.
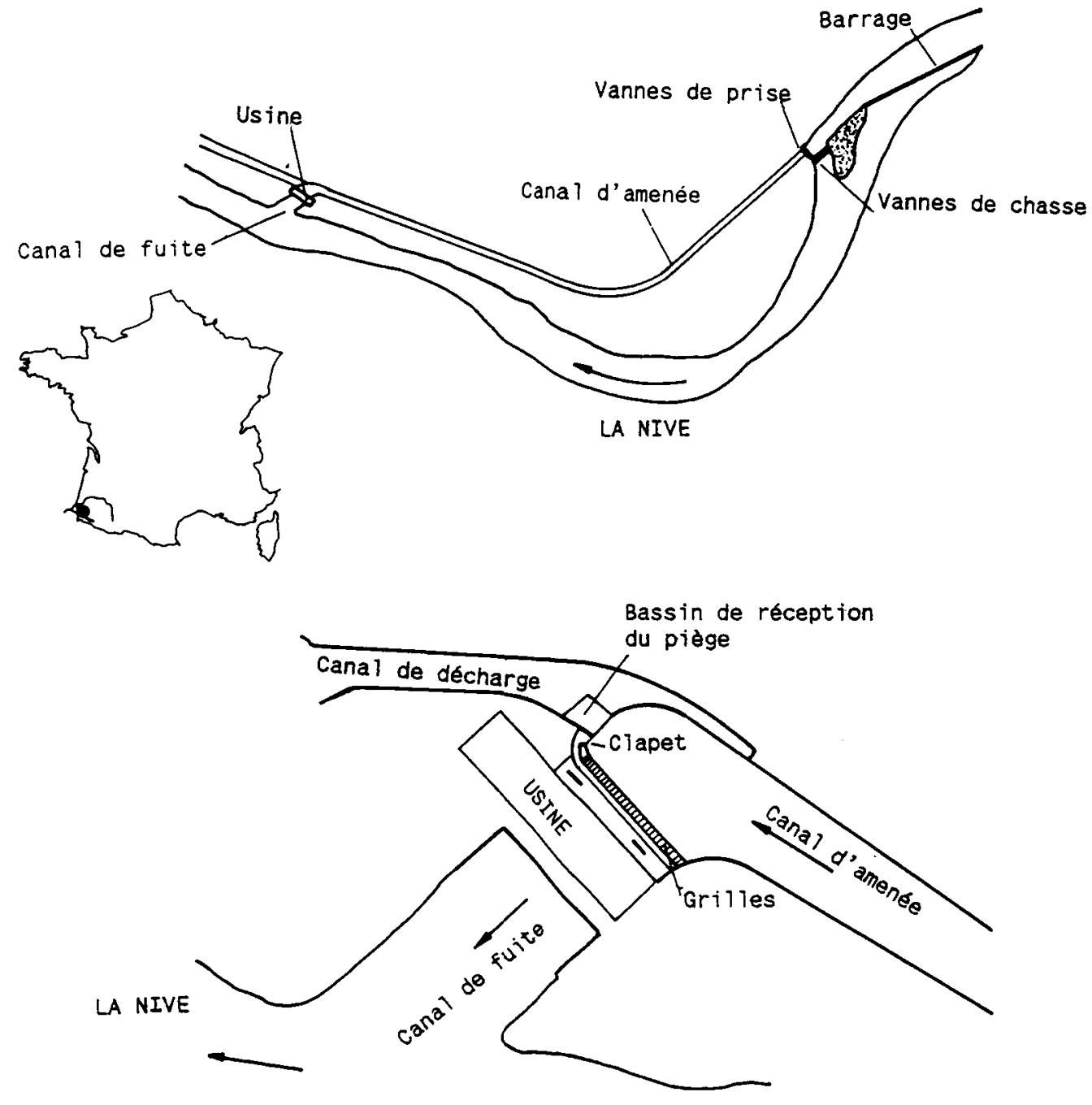

Figure 1 : Situation du site expérimental.

Figure 1 : Experimental site location. 


\subsection{Suivi de l'activité de dévalaison : piégeage et observation vidéo}

Le piège de dévalaison (fig. 2) est constitué d'une grille à barreaux de section carrée (clair entre barreaux $1 \mathrm{~cm}$ ) inclinée à $25 \%$, de $0.90 \mathrm{~m}$ de largeur et $1.50 \mathrm{~m}$ de longueur. Cette grille guide les poissons dans une goulotte (demi-tube PVC de $20 \mathrm{~cm}$ de diamètre) et permet de les capturer dans un vivier de $1.50 \mathrm{~m} \times 1.00 \mathrm{~m} \times 0.80 \mathrm{~m}$.

Le piège a été relevé systématiquement toutes les quatre heures pendant toute la durée des expérimentations de 1987 et 1988.

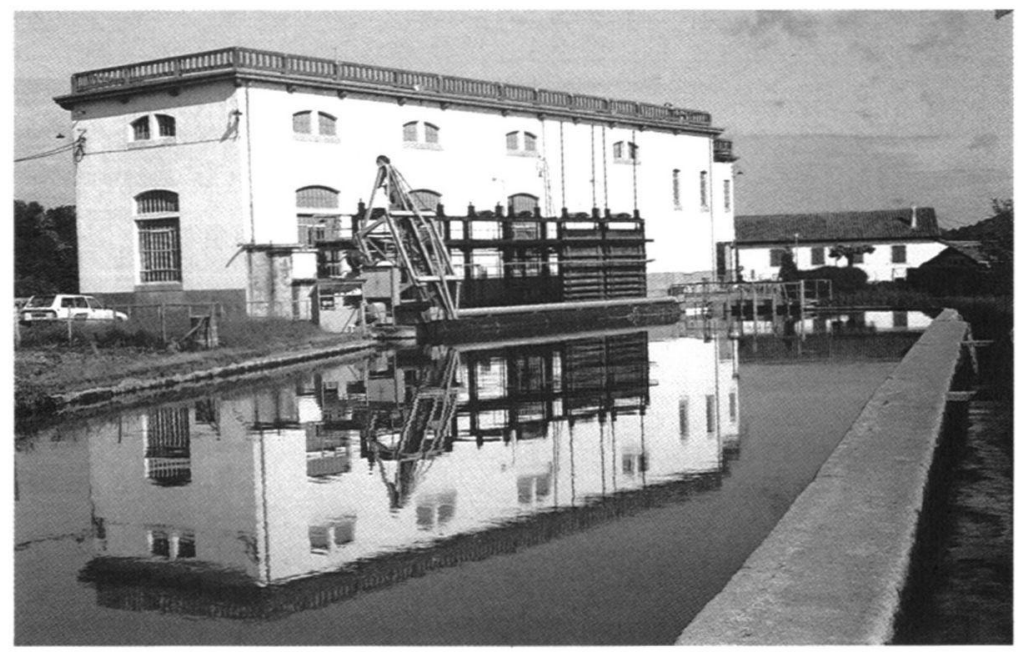

Photo 1 : L'usine hydroélectrique d'Halsou.

Photo 1 : A view of the Halsou powerhouse.

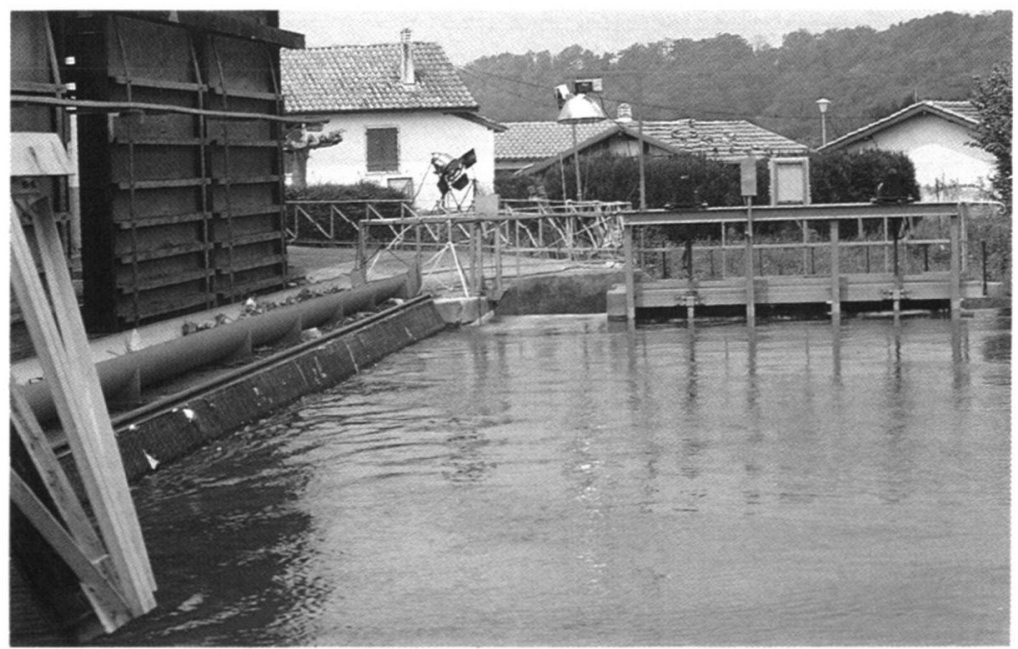

Photo 2 : Grilles de la centrale et exutoire de dévalaison (vue de l'amont).

Photo 2 : A view of the powerhouse trashrack and of the fish bypass looking downstream. 
Lors des expérimentations de 1987, un système vidéo de surveillance (caméra vidéo 50 Philips, magnétoscope PANASONIC NV 8051 à vitesse d'enregistrement variable), enregistrant en continu de $20 \mathrm{~h}$ à $8 \mathrm{~h}$, a permis de suivre la nuit le comportement des smolts au niveau du clapet.

En 1988, le système vidéo a été déplacé au niveau de la goulotte de piégeage pour faciliter les comptages, et les enregistrements ont été effectués en continu de jour comme de nuit.

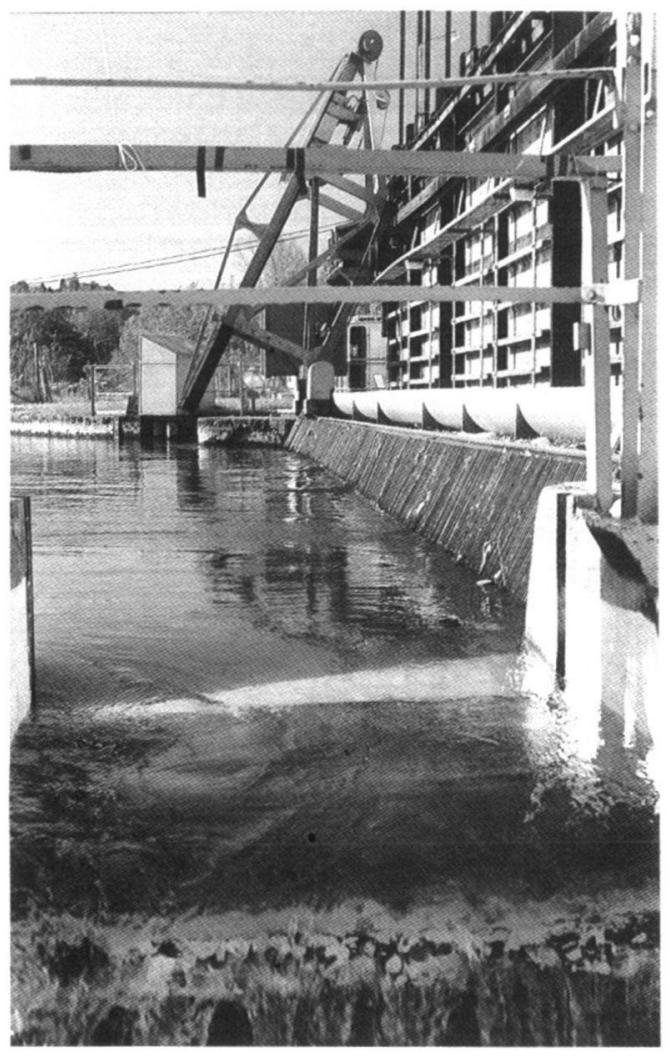

\section{Photo 3 : Exutoire de dévalaison et grilles de la centrale (vue de l'aval).}

Photo 3: A view of the fish bypass and of the powerhouse trashrack looking upstream.

\subsection{Paramètres du milieu}

Température de l'eau, teneur en oxygène dissous et conductivité ont été enregistrées en continu pendant toute la durée de la campagne 1987, le pH n'étant relevé que toutes les quatre heures. Ce même rythme d'enregistrement par période de quatre heures a été adopté pour l'ensemble de ces paramètres lors des expérimentations de 1988. La transparence de l'eau a fait l'objet de relevés quotidiens au disque de Secchi pendant les deux campagnes. Pression atmosphérique, précipitations, température de l'air et durée d'ensoleillement ont été obtenues auprès de la station météorologique de Biarritz. Les débits turbinés et débits de la Nive ont été fournis localement par l'usine E.D.F. 

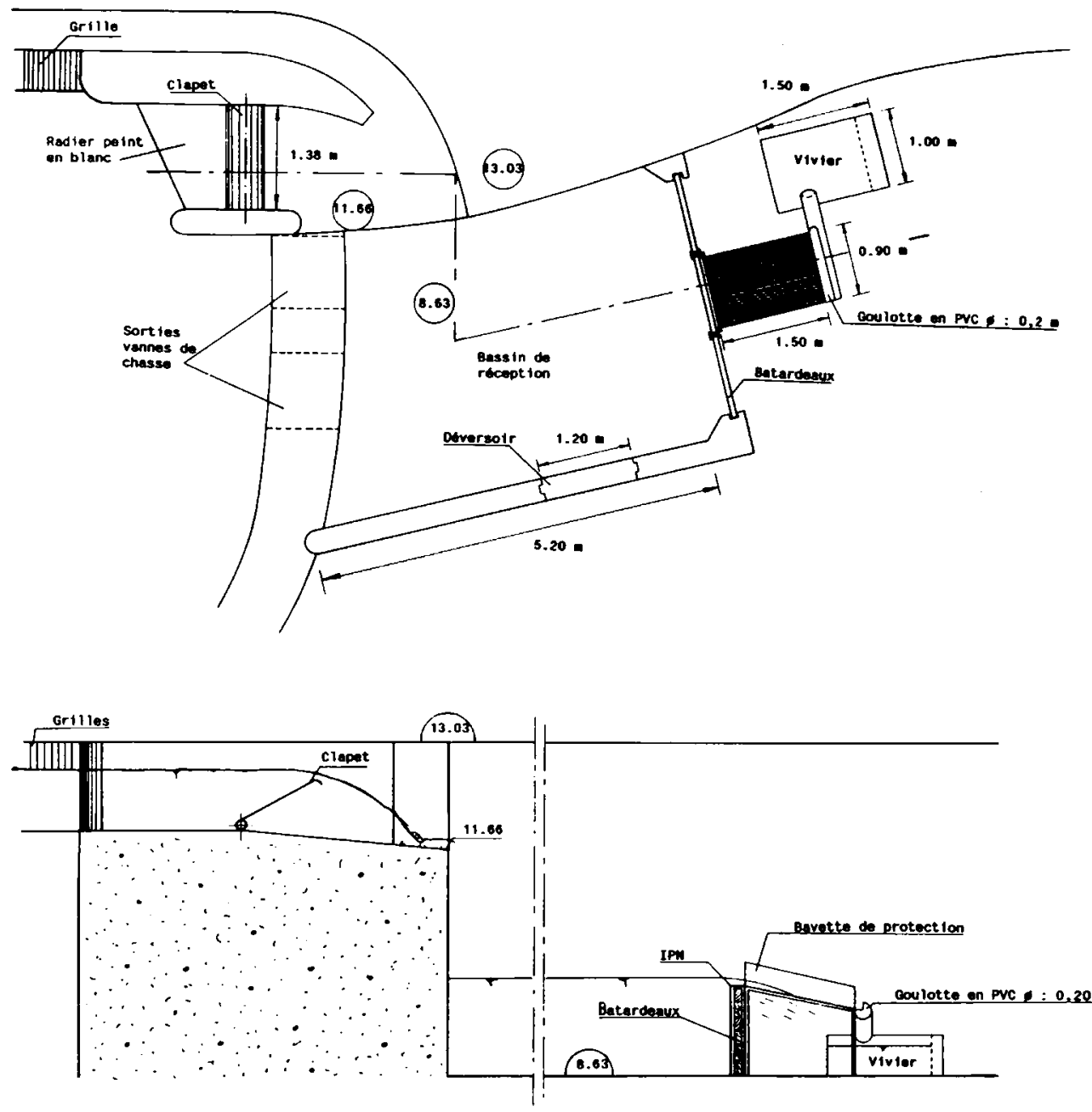

Figure 2 : Exutoire de dévalaison et dispositif de piégeage.

Figure 2 : Downstream migration bypass structure and trapping facilities.

\subsection{Débits au clapet}

Trois débits ont été testés au clapet de dévalaison : $100 \mathrm{I} / \mathrm{s}, 300 \mathrm{I} / \mathrm{s}$ et $500 \mathrm{I} / \mathrm{s}$. Les fluctuations du niveau dans le canal d'amenée (d'une amplitude d'une trentaine de centimètres hors période de crue) étant par moment très fréquentes et l'hydrodynamique du clapet complexe (existence de décollements, présence du radier à l'amont du clapet, position de la section de contrôle variable), ces valeurs ne sont que des ordres de grandeur du débit ayant effectivement transité par le clapet.

Chacun de ces débits a été testé sur un intervalle de temps correspondant à celui séparant deux relevés de piége consécutifs, c'est-à-dire 4 heures.

En 1987, chaque débit a été expérimenté 60 fois, dont 10 fois sur une même période horaire. En 1988, chaque débit a été expérimenté 84 fois, dont 14 fois sur une même tranche horaire.

\subsection{Lumières et activité de dévalaison nocturne}

Afin de juger des possibilités offertes par la lumière pour attirer et guider le poisson 
vers un exutoire de dévalaison, des tests ont été réalisés à titre exploratoire avec des projecteurs de qualité et d'intensité lumineuse différentes.

En 1987, les tests ont porté sur 2 types d'éclairage : un projecteur halogène de $2000 \mathrm{~W}$ et un projecteur à infrarouge (longueur d'onde $780 \mathrm{~nm}$ ) de $250 \mathrm{~W}$, orientés de façon à éclairer la zone d'influence du clapet. Cette zone a été éclairée une nuit sur deux avec le projecteur de $2000 \mathrm{~W}$ alors que le projecteur infrarouge permettait l'enregistrement vidéo le reste du temps.

En 1988, un projecteur halogène, deux lampes fluorescentes à vapeur de mercure et deux projecteurs infrarouge ont été utilisés. Le projecteur halogène de $2000 \mathrm{~W}$ a été placé à l'aplomb de la zone d'influence du clapet. Les deux lampes à vapeur de mercure de 400 W chacune ont été orientées de telle sorte que l'une éclaire la zone d'influence du clapet et l'autre la zone amont au niveau des grilles de protection des turbines. Les deux projecteurs infrarouge, de $500 \mathrm{~W}$ chacun, ont été installés pour couvrir la grille et la goulotte du piège de dévalaison, de manière à faciliter les comptages à la caméra vidéo.

La lampe halogène est une lampe classique à incandescence tungstène halogène pour prise de vue photographique $(2000 \mathrm{~W})$. Les lampes à vapeur de mercure utilisées sont des lampes à haute pression (HPL-N) à ballon fluorescent produisant de la lumière avec des pics d'émission proches de $400 \mathrm{~nm}, 430 \mathrm{~nm}$ (violet), $540 \mathrm{~nm}-560 \mathrm{~nm}$ (vert), 580-600 nm (jaune) et $620 \mathrm{~nm}$ (orange).

Deux programmateurs permettent d'alterner les éclairages dans la zone d'influence du clapet. La séquence lumière à vapeur de mercure/lumière halogène/obscurité $(\mathrm{V} / \mathrm{H} / \mathrm{O})$ peut ainsi être répétée.

En 1988, ces trois conditions d'éclairage ont été testées successivement sur des cycles d'une durée de 120 minutes, chaque type d'éclairage fonctionnant pendant 40 minutes. Une même séquence (correspondant à l'allumage d'une lampe donnée à une heure donnée) sur les six possibles (VHO, VOH, HVO, HOV, OVH, OHV) a été adoptée et répétée six fois chaque nuit, chacune des six séquences possibles ayant été par la suite répétée 6 fois sur 36 jours.

Chaque combinaison débit clapet/type d'éclairage $(100 \mathrm{l} / \mathrm{s}, 300 \mathrm{I} / \mathrm{s}$ ou $500 \mathrm{I} / \mathrm{s}$ avec $\mathrm{V}, \mathrm{H}$ ou O) a donc été testée 2 fois chaque jour entre $20 \mathrm{~h}$ et $8 \mathrm{~h}$ sur cette même période de 36 jours.

Indépendamment du débit et de la tranche horaire, il a été effectué 216 tests de $40 \mathrm{mn}$ pour chaque type d'éclairage.

\section{RÉSULTATS}

\subsection{Paramètres du milieu}

Les valeurs des principaux paramètres du milieu relevés lors de la campagne ont été portés à la figure 3 .

Lors de la campagne 1987, les débits ont varié de $14 \mathrm{~m}^{3 / \mathrm{s}}$ à $145 \mathrm{~m}^{3 / \mathrm{s}}$. La crue enregistrée en début de période de migration (6-16 avril) a rendu impossible l'utilisation du piège de dévalaison. La température de l'eau a varié sur l'ensemble de la campagne entre $10{ }^{\circ} \mathrm{C}$ et $17.3^{\circ} \mathrm{C}$.

Sur les 40 journées d'expérimentation, 19 ont été marquées par des précipitations plus ou moins abondantes avec un maximum de $19 \mathrm{~mm}$ le 11 avril. La pression atmosphérique a évolué entre $1002 \mathrm{hPa}$ et $1022 \mathrm{hPa}$. La Nive, hors des périodes de crue, est une rivière très peu turbide, sa transparence est généralement élevée, la moyenne étant voisine de $3.45 \mathrm{~m}$; cette transparence sur l'ensemble de la campagne a varié de plus de $4 \mathrm{~m}$ à $0.20 \mathrm{~m}$ lors d'une crue.

Les expérimentations de 1988 ont été marquées par des crues répétées de la Nive consécutives à des précipitations abondantes et de violents orages sur le bassin versant. Au total, 5 crues se sont succédées, conduisant à l'interruption du piégeage pendant 

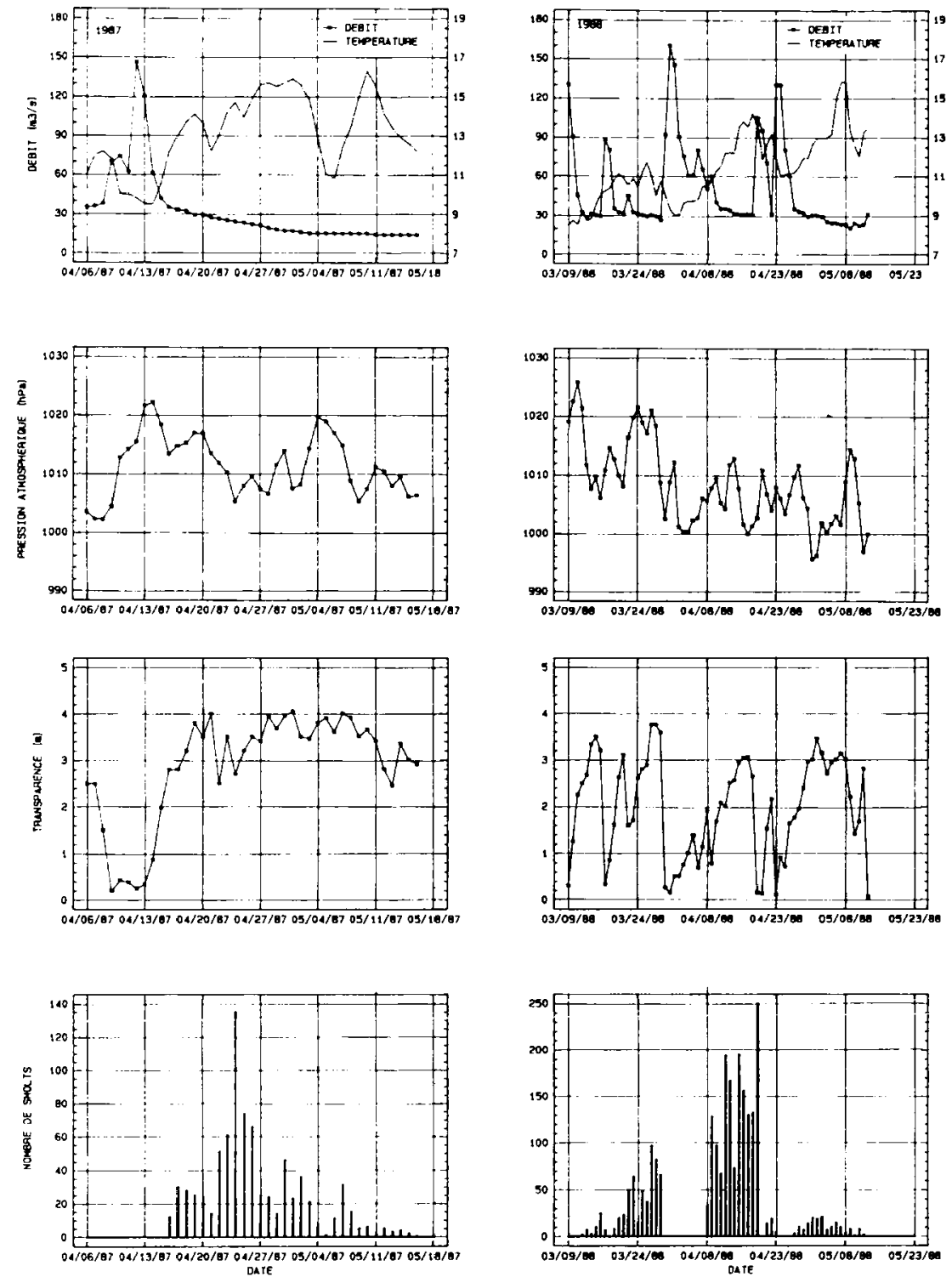

Figure 3 : Principaux paramètres du milieu et captures quotidiennes de smolts.

Figure 3 : Main environmental parameters and daily smolts catches.

plusieurs périodes d'une durée variant de un à neuf jours. La crue la plus importante s'est amorcée dans la nuit du 30 mars et s'est prolongée jusqu'au 8 avril (débit maximum $160 \mathrm{~m}^{3} / \mathrm{s}$ le 31 mars). Sur l'ensemble de la période, les débits ont variés de $25 \mathrm{~m}^{3 / \mathrm{s}}$ à $160 \mathrm{~m}^{3 / \mathrm{s}}$.

Sur l'ensemble de la campagne, la température moyenne journalière de l'eau a variée de $8.5{ }^{\circ} \mathrm{C}$ à $15.8{ }^{\circ} \mathrm{C}$.

Les précinitatinns farticulièrement abondantes ont été marquées par trois maxima nn+rables : $30 \mathrm{~mm}$ d'eau tombés en 24 heures le 29 mars (dont $75 \%$ en moins de 12 . _ules), environ $28 \mathrm{~mm}$ jans les journées du 19 avril et du 10 mai. Sur les 66 journées de campagne, 41 orı eté marquées par des chutes de pluie.

Lors de cette campagne, 5 dépressions ont été relevées. Les plus importantes ont 
été enregistrées le 17 avril, et les $1^{\text {er }}$ et 2 mai, avec des minima voisins de $995 \mathrm{hPa}$. Sur l'ensemble des expérimentations, la pression atmosphérique a varié entre $995 \mathrm{hPa}$ et $1026 \mathrm{hPa}$.

La transparence de l'eau a varié de $0.05 \mathrm{~m}$ le 13 mai à $3.75 \mathrm{~m}$ les 27 et 28 mars. La transparence moyenne enregistrée en 1988, plus faible que celle observée en 1987, a été voisine de $2 \mathrm{~m}$.

\subsection{Hydraulique de l'exutoire de dévalaison}

De façon schématique, on a pu distinguer trois conditions hydrauliques au niveau de l'exutoire de dévalaison, fonction du débit transitant par le clapet et dans une moindre mesure du niveau d'eau dans le canal d'amenée (figure 4). Pour les débits les plus faibles (en particulier $100 \mathrm{l} / \mathrm{s}$ ), la section de contrôle se trouve au niveau de la crête déversante
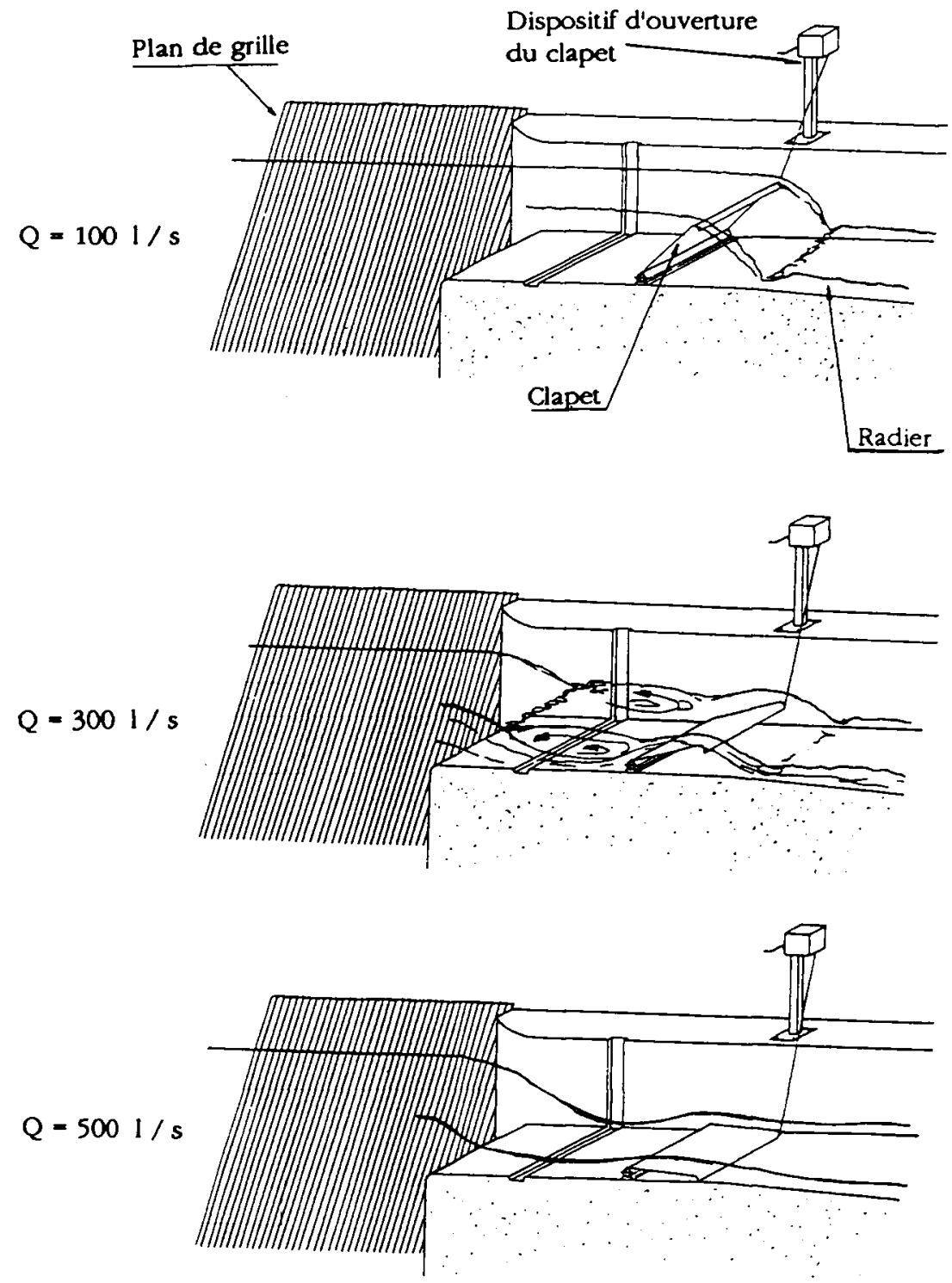

Figure 4 : Conditions hydrauliques dans l'exutoire de dévalaison.

Figure 4 : Hydraulic conditions in the fish bypass structure. 
du clapet qui se comporte comme un déversoir mince. Les conditions hydrauliques en amont sont relativement homogènes, les décollements au niveau de l'entonnement restent peu marqués et les vitesses d'approche faibles.

Lorsque le clapet est fortement abaissé, en particulier pour un débit de $500 \mathrm{l} / \mathrm{s}$, la section de contrôle remonte vers l'amont au niveau du rétrécissement, le système fonctionne alors en déversoir épais.

Entre ces deux configurations d'écoulement très différentes, on observe une forme intermédiaire : l'écoulement s'accélère et passe en torrentiel au niveau du rétrécissement puis repasse en fluvial à l'amont du clapet avec formation d'un ressaut plus ou moins marqué en surface, plus net sur la partie droite de l'exutoire. Un décollement très prononcé se forme le long de la paroi située en rive droite de l'exutoire.

Un certain nombre de mesures de vitesses ont été effectuées au micro-moulinet au niveau de l'exutoire de dévalaison. Lorsque la profondeur le permettait, ces mesures ont été prises à des distances de $0.2 \mathrm{~h}, 0.4 \mathrm{~h}$ et $0.8 \mathrm{~h}$ (h étant le tirant d'eau) à partir du fond, ainsi qu'à la surface.

Pour les cotes amont les plus fréquemment rencontrées lors de la campagne 1988 (12.70-12.80), les vitesses moyennes mesurées au niveau de l'entonnement du clapet varient de $0.20 \mathrm{~m} / \mathrm{s}$ à $0.30 \mathrm{~m} / \mathrm{s}$ pour un débit de $100 \mathrm{I} / \mathrm{s}$ et atteignent $0.70 \mathrm{~m} / \mathrm{s}$ à $0.95 \mathrm{~m} / \mathrm{s}$ pour un débit de $500 \mathrm{l} / \mathrm{s}$.

Il est évident que la valeur de cette vitesse est fortement liée à la cote du plan d'eau : pour un débit clapet de $300 \mathrm{l} / \mathrm{s}$, la vitesse moyenne passe de $0.50-0.75 \mathrm{~m} / \mathrm{s}$ à 1.30 $1.50 \mathrm{~m} / \mathrm{s}$ lorsque le niveau d'eau s'abaisse d'une trentaine de centimètres.

La cote du plan d'eau amont fluctuant suivant le débit dans la Nive et celui absorbé par les turbines, les conditions de vitesses à l'amont immédiat du clapet se sont révélées très changeantes pour un même débit du clapet.

Des mesures de vitesse en surface (à $0.05 \mathrm{~m}$ et à $0.50 \mathrm{~m}$ de la surface) ont également été effectuées au niveau des grilles de la centrale lorsque les trois turbines sont en fonctionnement. Elles varient pour la plupart entre $0.40 \mathrm{~m} / \mathrm{s}$ et $0.75 \mathrm{~m} / \mathrm{s}$, leur moyenne étant voisine de $0.60 \mathrm{~m} / \mathrm{s}(\sigma=0.141)$. Les vitesses normales mesurées dans la zone située à proximité du clapet sont significativement plus faibles (entre $0.20 \mathrm{~m} / \mathrm{s}$ et $0.50 \mathrm{~m} / \mathrm{s}$ ).

La composante de la vitesse parallèle aux grilles ("vitesse de balayage" ou "vitesse de transport" en utilisant la terminologie propre aux dispositifs de guidage) a été grossièrement évaluée lorsque les trois turbines étaient en fonctionnement. Ces vitesses de balayage, orientées en direction du clapet, ont une valeur moyenne voisine de $0.70 \mathrm{~m} / \mathrm{s}$ $(\sigma=0.21)$. Elles varient de $0.30 \mathrm{~m} / \mathrm{s}$ à un peu plus de $1 \mathrm{~m} / \mathrm{s}$. Comme les vitesses normales, les vitesses de balayage diminuent de façon significative à proximité du clapet.

En rive droite, le décrochement dans le mur entre le plan de grille et l'entonnement du clapet génère un courant de retour : les vitesses de balayage diminuent brusquement d'intensité puis changent de sens.

\subsection{Suivi de l'activité de dévalaison}

\subsubsection{Activité quotidienne (fig. 3)}

Près de 800 smolts ont été capturés dans le piège du 16 avril au 16 mai $1987.48 \%$ des captures ont été effectuées entre le 22 et le 26 avril. II est à noter que ce nombre relativement modéré doit correspondre à la fin de la migration, la plus grosse partie de la population ayant dû dévaler lors des crues précédant la période d'observation.

Plus de 2400 smolts ont été capturés dans le piège de dévalaison entre le 9 mars et le 13 mai 1988. Deux périodes de forte activité migratoire ont été observées. La première, située entre le 13 et le 30 mars, a vu dévaler environ $23.5 \%$ des smolts piégés au cours de la campagne. Le plegeage a dû être interrompu suite à une montée des eaux alors que

. etait encore notable. La seconde, comprise entre le 8 et le 19 avril, englobe le pic migratoire (271 c...ults le 19 avril, dont 176 capturés en moins de 4 heures) et représente $68.3 \%$ des captures. Le piégeage a dû être interrompu suite à une augmentation brutale du débit alors que l'activité de dévalaison semblait être à son maximum. 
Bull. Fr. Pêche Piscic. (1991) $321: 72-92 \quad-82-$

\subsubsection{Activité nycthémérale}

En 1987, $78.6 \%$ des smolts ont dévalé le jour contre $21.4 \%$ la nuit. La différence est hautement significative $\left(F=10.7 ; d d l=1,50 ; p=0.001^{\star \star \star}\right)$. Les pourcentages dévalant la journée varient de $51.8 \%$ à $100 \%$. Si l'on fait les moyennes hebdomadaires sur les quatre semaines de l'expérimentation, le pourcentage de dévalaison diurne varie de $68 \%$ à plus de $80 \%$.

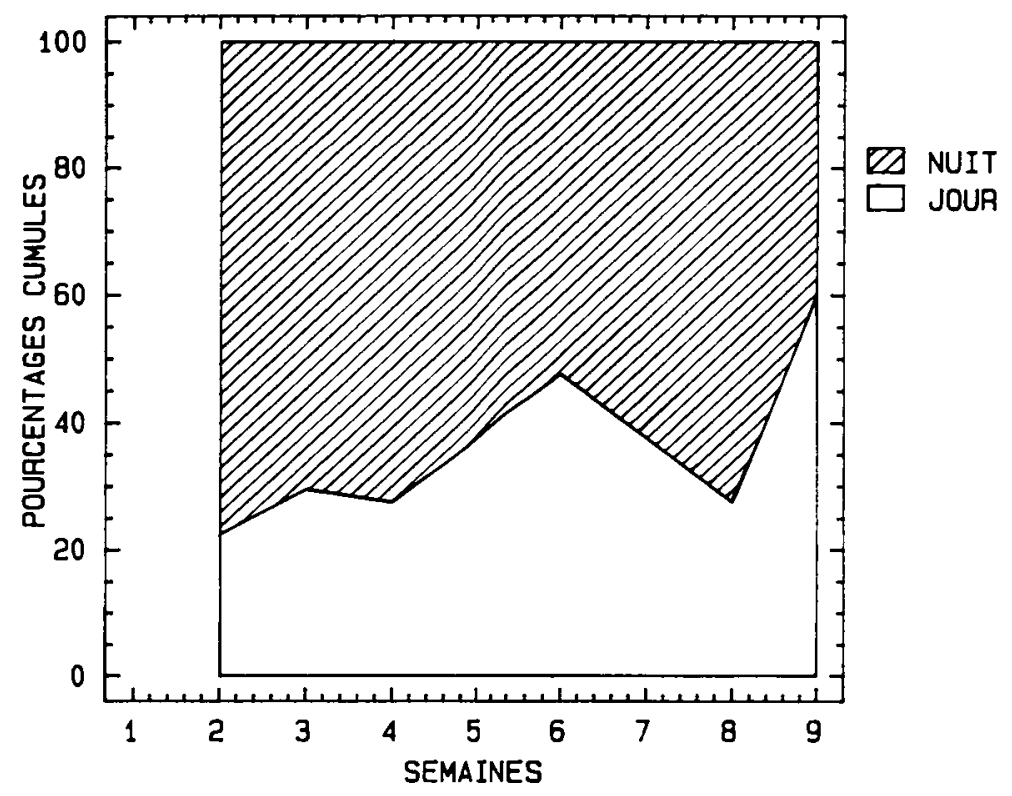

Figure 5 : Evolution des passages diurnes en 1988.

Figure 5 : Evolution of diurnal passages in 1988.

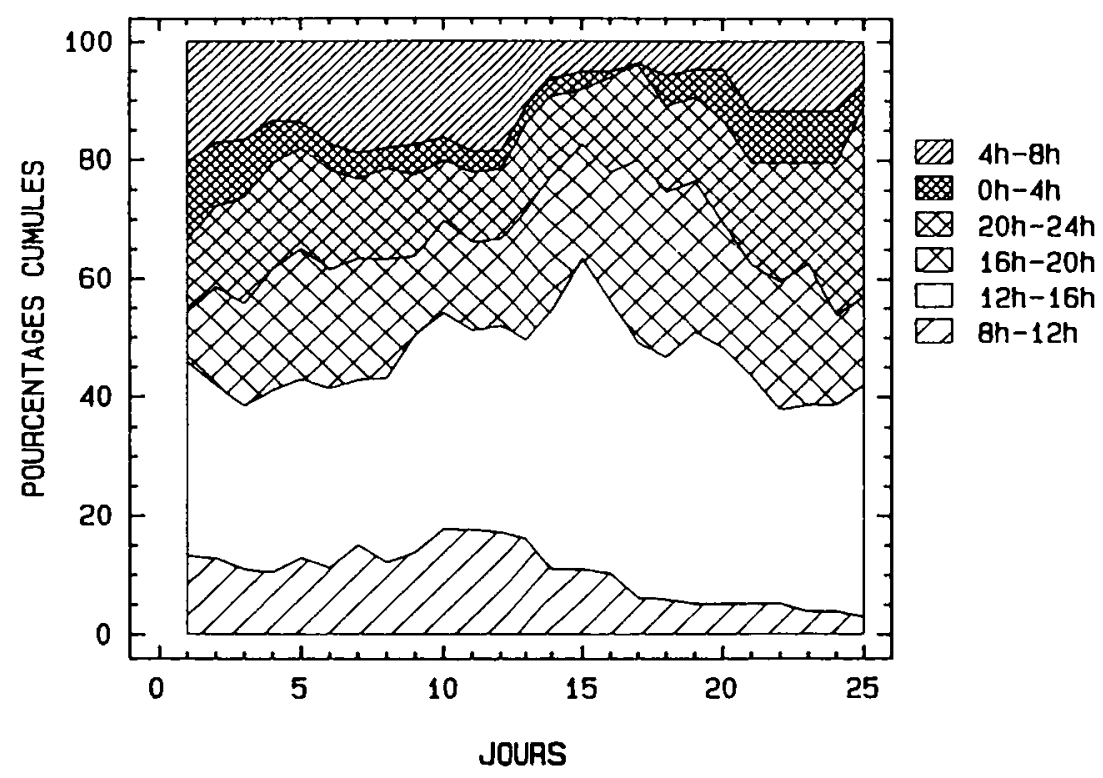

Figure 6 : Nombre de smolts piégés par intervalle de $4 \mathrm{~h}$ en 1987.

Figure 6 : Catch of smolts by 4 h-period in 1987. 
En $1988,62.4 \%$ des smolts capturés ont dévalé la nuit contre $37.6 \%$ le jour. On observe cependant des différences sensibles d'activité entre le début et la fin de la migration. La figure 5, sur laquelle ont été portés les pourcentages moyens hebdomadaires de smolts piégés le jour et la nuit, montre l'évolution du rythme nycthéméral au cours de la campagne. La dévalaison est essentiellement nocturne en début de période (plus de $70 \%$ ), la tendance s'inversant en fin de migration : lors de la neuvième semaine, $40 \%$ des passages sont enregistrés la nuit contre $60 \%$ le jour.

\subsubsection{Activité horaire}

Le rythme de dévalaison a d'abord été examiné par intervalles de quatre heures correspondant à la période des relevés successifs du piège. En 1987, il apparaît une différence significative entre les différentes tranches $\left(F=4.6 ; d d l=1,50 ; p=0.036^{*}\right)$, le pic migratoire se situant en début d'après-midi dans la tranche 12 h-16 h et le "creux" se situant dans la tranche $8 \mathrm{~h}-12 \mathrm{~h}$. On a porté sur la figure 6 l'évolution du pourcentage de smolts dévalant entre chaque relevé de piège : la série des pourcentages a été lissée par moyenne mobile calculée sur sept jours consécutifs. Ce lissage ne met en évidence aucune tendance nette d'évolution du rythme de dévalaison sur la période considérée. La période étudiée ne concerne cependant que la fin de la migration.

En 1988, le rythme de dévalaison a été examiné par intervalles de quatre heures correspondant aux relevés successifs du piège (fig. 7). L'analyse de variance montre des différences statistiquement significatives d'activité entre les différentes périodes étudiées $\left(F=2.2 ; d d l=5,312 ; p=0.05^{*}\right)$. L'activité de plus forte dévalaison a été enregistrée entre $20 \mathrm{~h}$ et $24 \mathrm{~h}(28.3 \%)$ alors que la plus faible se situe entre $8 \mathrm{~h}$ et $12 \mathrm{~h}(7.7 \%)$.

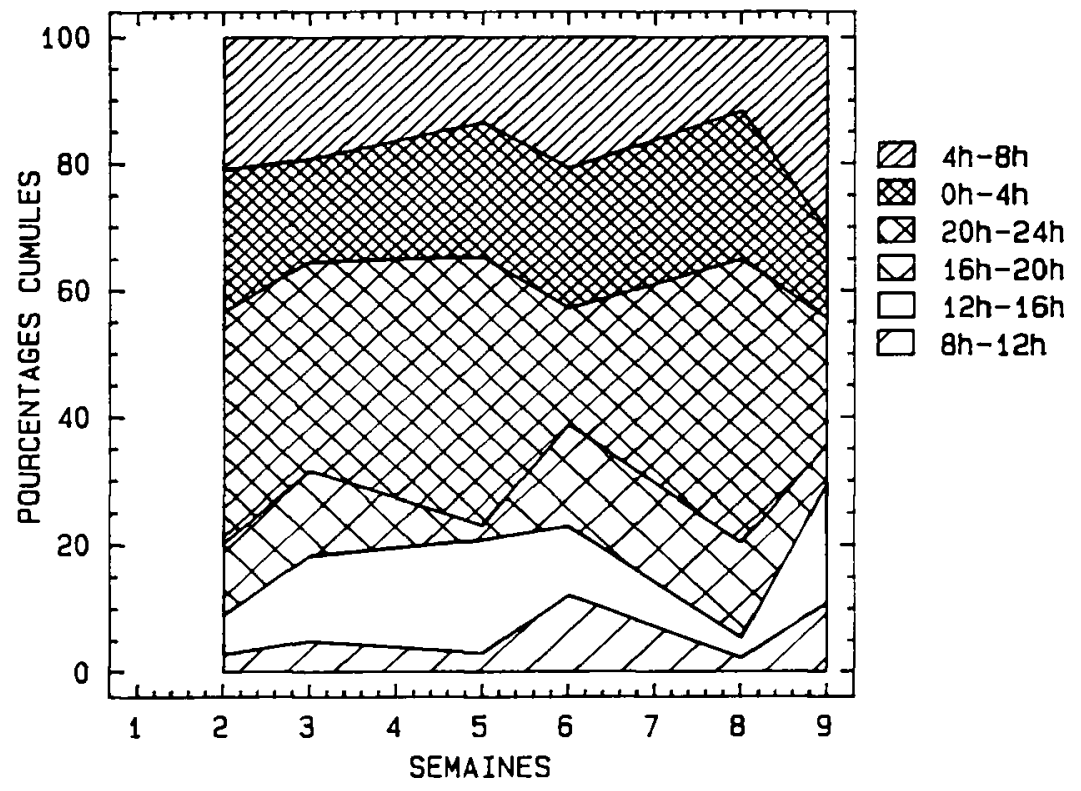

Figure 7 : Nombre de smolts piégés par intervalle de $4 \mathrm{~h}$ en 1988.

Figure 7 : Catch of smolts by 4 h-period en 1988.

Cette même année, les passages ont été comptabilisés plus finement par tranche horaire sur l'ensemble de la période d'expérimentation grâce au système vidéo. L'analyse des enregistrements montre que les pics horaires de smolts piégés se situent entre 22 h et $24 \mathrm{~h}$ (fig. 8). A l'opposé, le "creux" de l'activité de dévalaison se situe entre $9 \mathrm{~h}$ et $13 \mathrm{~h}$ puis entre 16 h et $18 \mathrm{~h}$. 


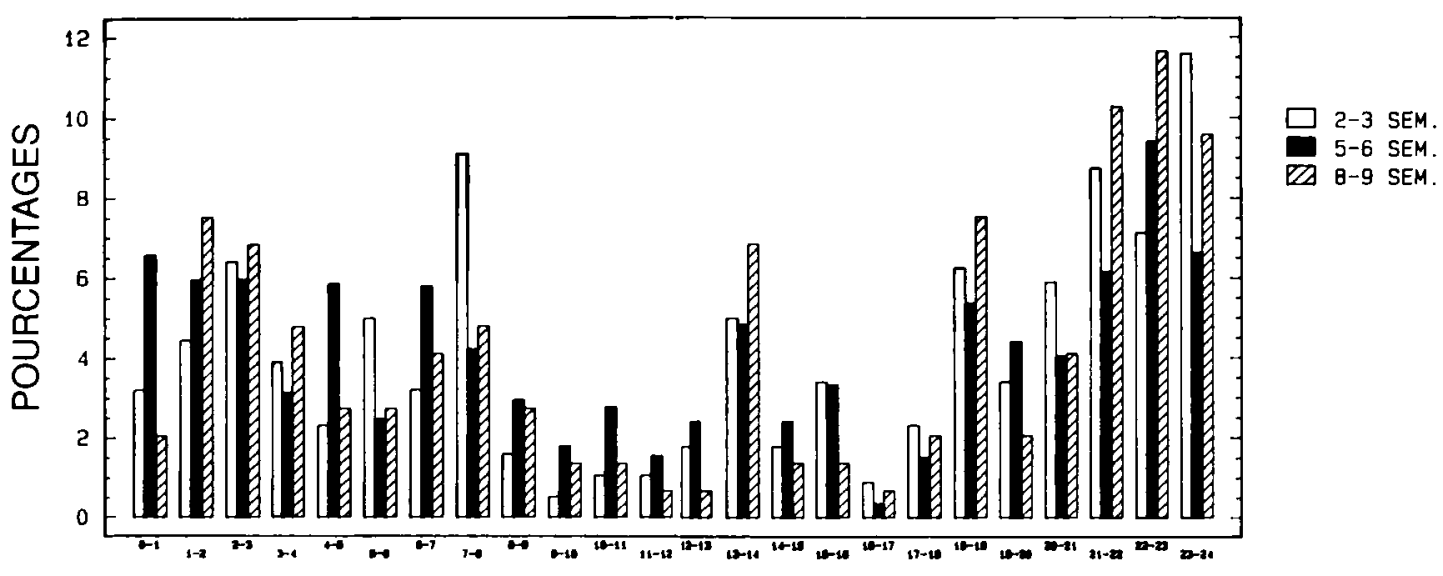

Figure 8 : Rythme horaire des passages à l'exutoire (1988).

Figure 8 : Hourly passages through bypass by video recording (1988).
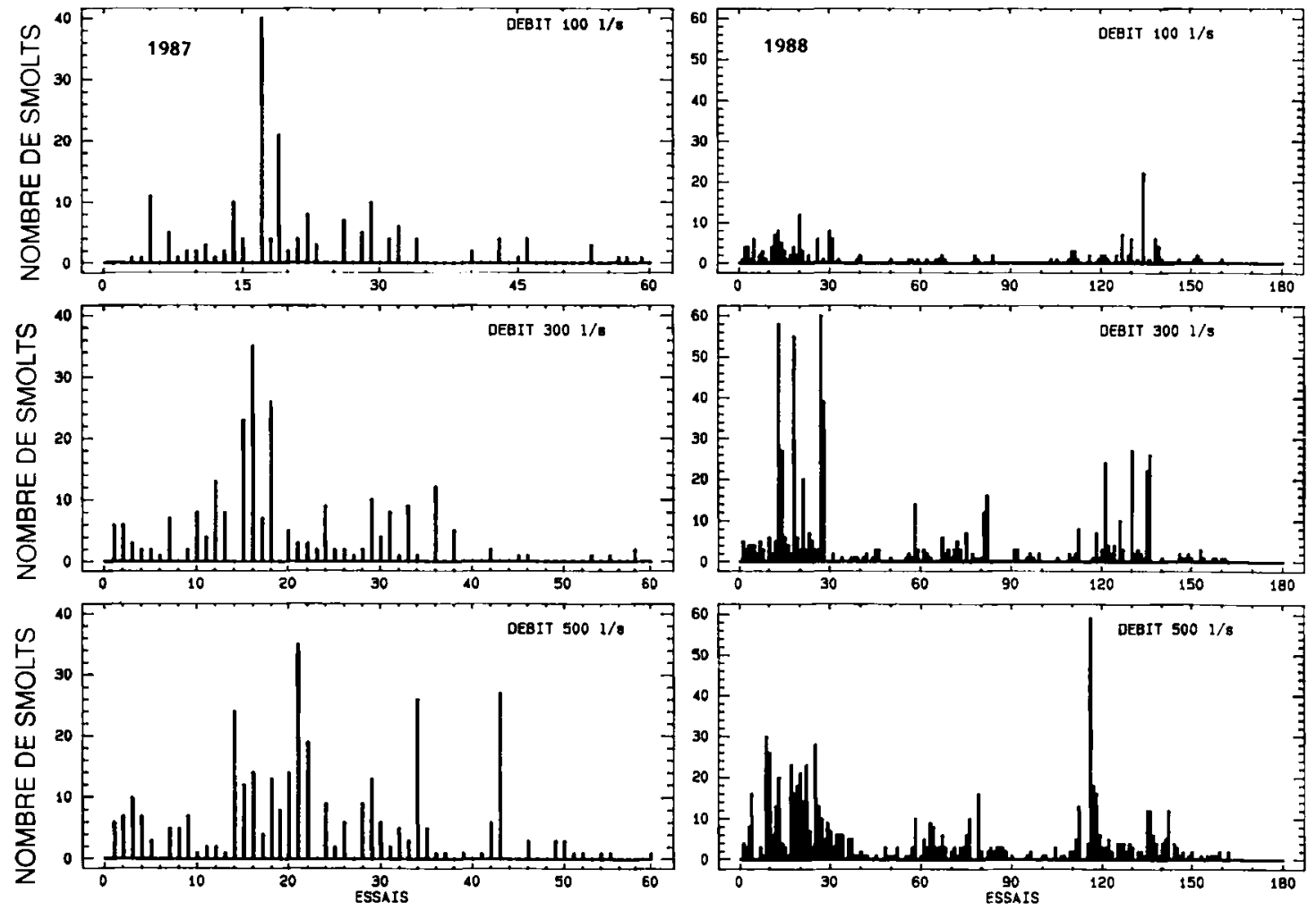

Figure 9 : Influence du débit sur les passages de smolts dans l'exutoire (1987 et 1988).

Figure 9 : Smolt passages related to bypass discharge (1987 and 1988). 


\subsection{Influence du débit du clapet de dévalaison}

On rappelle que trois débits ont été testés : $100 \mathrm{l} / \mathrm{s}, 300 \mathrm{l} / \mathrm{s}$ et $500 \mathrm{l} / \mathrm{s}$, chacun d'entre eux étant testé sur un intervalle de temps correspondant à celui séparant deux relevés du piège consécutifs.

On a porté sur la figure 9 le nombre de smolts dévalant suivant les différentes valeurs du débit transitant par le clapet.

En 1987, si on prend en compte l'ensemble des données, on observe que le nombre de poissons transitant par le clapet augmente sensiblement avec le débit $(100 \mathrm{l} / \mathrm{s}, 300 \mathrm{I} / \mathrm{s}$ et $500 \mathrm{l} / \mathrm{s}$ ) mais les différences ne sont pas significatives ( $F=1.0 ; d d l=2,177 ; p=0.36)$. Une moyenne de 3.6 smolts $/ 4 \mathrm{H}$ (smolts par période de 4 heures) a dévalé avec $100 \mathrm{l} / \mathrm{s}$ $(\sigma=10.1), 4 \mathrm{smolts} / 4 \mathrm{H}$ avec $300 \mathrm{l} / \mathrm{s}(\sigma=6.6)$, et $5.6 \mathrm{smolts} / 4 \mathrm{H}$ avec $500 \mathrm{l} / \mathrm{s}(\sigma=7.6)$.

Par contre, si on enlève une seule observation correspondant au pic de migration observé, qui s'est produit lorsque le clapet était faiblement alimenté, la différence devient statistiquement discernable ( $\left.F=4.1 ; d d l=2,176 ; p=0.017^{\star}\right)$. Les moyennes sont alors pour $100 \mathrm{l} / \mathrm{s}, 300 \mathrm{l} / \mathrm{s}$ et $500 \mathrm{l} / \mathrm{s}$ respectivement de $2.3 \mathrm{smolts} / 4 \mathrm{H}, 3.4 \mathrm{smolts} / 4 \mathrm{H}$ et 5.4 smolts $/ 4 \mathrm{H}$.

Lors de la campagne 1988, une moyenne de 4.9 smolts $/ 4 \mathrm{H}$ a dévalé avec $100 \mathrm{I} / \mathrm{s}$ transitant par le clapet $(\sigma=1.12), 10 \mathrm{smolts} / 4 \mathrm{H}$ avec $300 \mathrm{l} / \mathrm{s}(\sigma=2.55)$ et $13.7 \mathrm{smolts} / 4 \mathrm{H}$ avec $500 \mathrm{l} / \mathrm{s}(\sigma=2.02)$. Au total, $17.1 \%$ des smolts capturés ont franchi le clapet avec un débit réglé à $100 \mathrm{l} / \mathrm{s}, 35 \%$ avec $300 \mathrm{I} / \mathrm{s}$ et $47.9 \%$ avec $500 \mathrm{I} / \mathrm{s}$. L'analyse de variance fait apparaître une différence très significative entre les nombres de smolts piégés ( $F=4.9$; $d d l=2,249 ; p=0.007^{\star \star}$ ) suivant les différents débits testés.

On a tenté de mettre en évidence plus finement l'influence du débit du clapet en fonction de "l'intensité" de l'activité de dévalaison , ou plus exactement du nombre de poissons piégés. Pour ce faire, trois classes d'abondance ont été définies en fonction du nombre de captures enregistrées quotidiennement au piège. Les épisodes correspondant aux classes 10-70 smolts/jour, 71-130 smolts/jour et supérieurs à 130 smolts/jour ont été chacun soumis à une analyse de variance pour les trois débits testés dans le clapet.

Pour les deux classes correspondant à $71-130$ smolts/jour et $>130$ smolts/jour, if n'apparaît aucune différence significative entre les trois débits transitant au clapet (respectivement $F=2.8 ; d d=2,30 ; p=0.073$ et $F=1.4 ; d d l=2,30 ; p=0.26$ ).

Toutefois, lorsque seuls les débits $100 \mathrm{l} / \mathrm{s}$ et $500 \mathrm{l} / \mathrm{s}$ sont pris en compte, il apparaît une différence hautement significative $\left(F=8.9 ; d d l=1,20 ; p=0.007^{* *}\right)$ et $(F=8.1 ; d d l=1,2$; $\left.p=0.009^{\star *}\right)$.

Par contre, pour la classe 10-70 smolts/jour correspondant aux plus faibles effectifs journaliers, il existe une différence hautement significative entre le nombre de smolts piégés suivant les trois débits considérés transitant par le clapet $\left(F=7.2 ; d d l=2,126 ; p=0.001^{\star \star \star}\right)$; cette différence est aussi significative pour certains débits traités deux à deux : entre $100 \mathrm{I} / \mathrm{s}$ et $300 \mathrm{l} / \mathrm{s}$, puis entre $100 \mathrm{l} / \mathrm{s}$ et $500 \mathrm{l} / \mathrm{s}$ (respectivement $F=11.6 ; d d l=1,84 ; p=0.001^{* \star \star}$ et $\left.\mathrm{F}=12.1 ; \mathrm{dd}=1,84 ; \mathrm{p}=0.000^{* * *}\right)$.

Ce résultat tendrait à montrer que le nombre de smolts piégés est lié à l'importance du débit transitant par le clapet, et ce de façon d'autant plus nette que les effectifs dévalant sont modérés.

N.B. : Les résultats des analyses de variance sont donnés sous la forme usuelle :

$$
\left(F=4.9 ; d d l=2,249 ; p=0.007^{\star *}\right)
$$

où $\mathrm{F}$ : le rapport de la variance expliquée à la variance inexpliquée

ddl : les degrés de liberté

$p$ : le seuil de signification

(") signifie une différence significative au seuil de signification de $5 \%$

(") signifie une différence très significative au seuil de signification de $1 \%$

("*) signifie une différence très hautement significative au seuil de signification de $0,1 \%$.

\section{Références}

- WANNACOTT T. ET WANNACOTT R. 1984. Statistiques. Economica, 790 p.

— DAGNELIE P. 1986. Théorie et méthodes statistiques, volume III. Presses Agronomiques de Gembloux, $461 p$. 


\subsection{Influence de la lumière sur la dévalaison nocturne}

\subsection{1. Éclairage du clapet}

En 1987, la zone d'influence du clapet a été éclairée une nuit sur deux avec un projecteur de 2000 watts, un projecteur infrarouge de 250 watts permettant l'enregistrement vidéo le reste du temps. II n'a été mis en évidence aucune influence significative de l'éclairage sur la migration : $51.2 \%$ des poissons ont.dévalé avec le projecteur halogène et $48.8 \%$ avec l'infrarouge ( $F=0.017 ; d d l=1,277 ; p=0.90)$.

En 1988, outre le projecteur halogène, deux lampes fluorescentes à vapeur de mercure ont été utilisées. Le projecteur halogène de $2000 \mathrm{~W}$ a été placé à l'aplomb de la zone d'influence du clapet, les deux lampes à vapeur de mercure de $400 \mathrm{~W}$ chacune ont été orientées de telle sorte que l'une éclaire la zone d'influence du clapet et l'autre la zone amont au niveau des grilles de la prise d'eau.

En période nocturne, il est descendu une moyenne de 5.2 smolts $/ 40 \mathrm{mn}(\sigma=9.85)$ lorsque le clapet était laissé dans l'obscurité, 3 smolts $/ 40 \mathrm{mn}(\sigma=6.60)$ pendant la phase d'éclairage avec le projecteur halogène et $1.5 \mathrm{smolt} / 40 \mathrm{mn}(\sigma=2.83)$ pendant la phase d'éclairage avec les lampes à vapeur de mercure. Les conditions de franchissement pour les juvéniles sont donc meilleures lorsque le clapet est laissé dans l'obscurité.

L'analyse de variance consistant à tester globalement les 3 types de lumière ordonnés selon les 3 conditions de débit transitant par le clapet montre une différence hautement significative à la fois entre les différents éclairages $(F=11.6$; ddl $=2,477$; $\left.p=0.000^{\star \star \star}\right)$ et les différents débits $\left(F=11.5 ; d d l=2.477 ; p=0.000^{\star \star \star}\right)$. Aucune interaction n'apparaît entre débits et lumière $(F=1.1 ; d d=4,477 ; p=0.34)$.

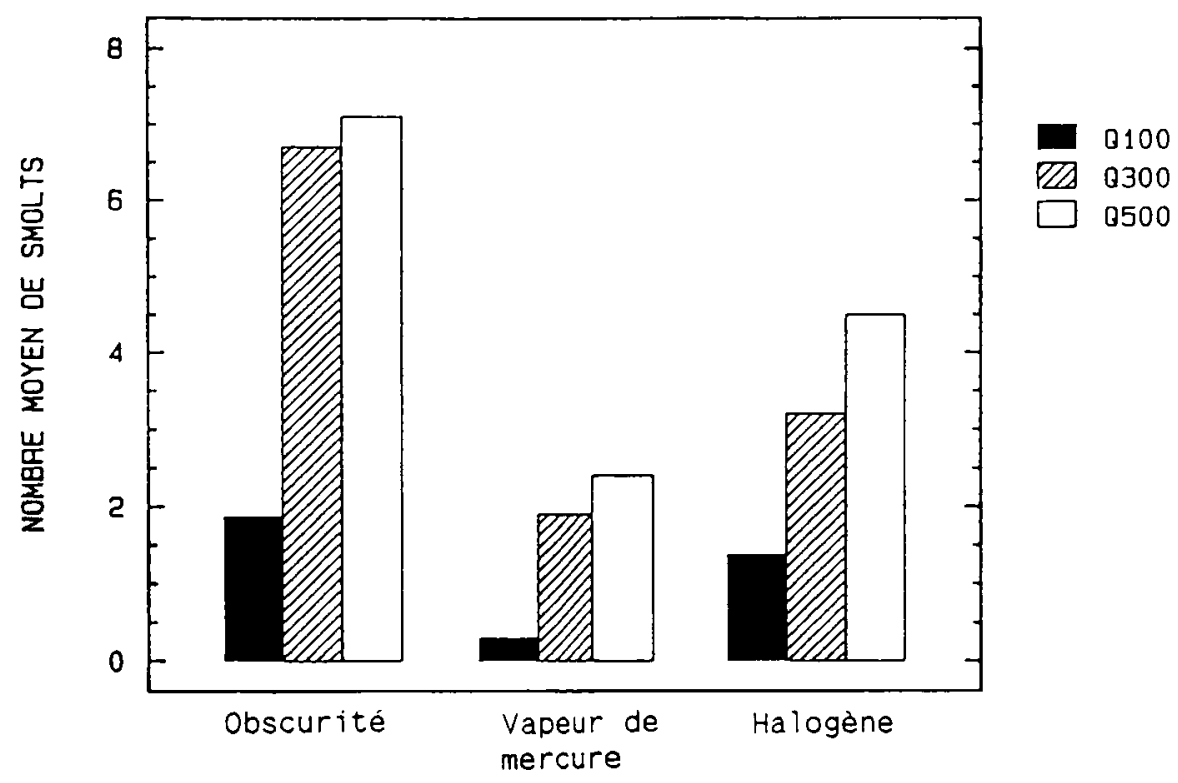

Figure 10 : Passages moyens de smolts (par période de $40 \mathrm{mn}$ ) en fonction du débit dans l'exutoire et des conditions d'éclairage.

Figure 10 : Mean smolts passages (by $40 \mathrm{mn}$-period) related to bypass discharge and light conditions. 
Les différents types d'éclairage agissent toujours de façon identique quels que soient les débits au clapet (fig. 10) : les smolts dévalent nettement mieux lorsque le clapet est maintenu dans l'obscurité que lorsqu'il est éclairé avec le projecteur halogène et surtout avec les lampes à vapeur de mercure, et ce, quel que soit le débit.

Les conditions hydrauliques rencontrées aux abords du clapet de dévalaison jouent donc un rôle prépondérant sur les passages des smolts : quelles que soient les conditions d'éclairage, il descend toujours moins de juvéniles à $100 \mathrm{l} / \mathrm{s}$ qu'à $300 \mathrm{l} / \mathrm{s}$ ou $500 \mathrm{l} / \mathrm{s}$.

\subsubsection{Observations complémentaires sur l'influence de la lumière}

Des expériences ont été effectuées dans le canal d'amenée avec des saumons adultes au cours desquelles la lampe à vapeur de mercure située le plus en amont du clapet a été allumée alors que le site était plongé dans l'obscurité complète. II a alors été constaté une réaction phototropique de la part des poissons qui semblaient être attirés vers la zone éclairée. Ils se maintenaient cependant toujours hors de la zone d'éclairement maximum. En balayant lentement la surface de l'eau avec la lampe, il était possible de faire se déplacer les saumons qui demeuraient cependant à la frange du halo lumineux. Des observations similaires, menées dans les mêmes conditions, ont été effectuées sur les smolts. Lorsque la lampe à vapeur de mercure située au-dessus de la zone d'influence du clapet était allumée, les smolts semblaient alors "jaillir" du fond du canal d'amenée pour venir évoluer à une profondeur intermédiaire (de l'ordre de $1.50 \mathrm{~m}$ ). Les rares individus qui remontaient trop près de la surface dans la zone fortement éclairée manifestaient une réaction immédiate d'éloignement.

\subsection{Contrôle de l'efficacité du clapet}

Le clapet s'étant révélé satisfaisant en tant que dispositif permettant le passage des smolts, il convenait d'en évaluer l'efficacité, c'est-à-dire de déterminer, sur le nombre de smolts empruntant le canal d'amenée, la proportion transitant par l'exutoire et celle passant par les turbines. Pour ce faire, 5 opérations de marquages et recaptures ont été effectuées hors période de crue (Tableau I).

Certains smolts capturés dans le piège ont été marqués par ablation légère de l'une des extrémités des nageoires caudales, dorsales ou pelviennes. L'opération de marquage terminée, les poissons ont été acheminés puis déversés soit en amont immédiat de la prise d'eau du canal d'amenée (station A), située environ à $1000 \mathrm{~m}$ de l'usine, soit au niveau de la station d'épuration de Cambo-les-Bains (station B) distante de $2500 \mathrm{~m}$ à $3000 \mathrm{~m}$ en amont de cette même usine.

Lors de la première opération de marquage effectuée le 10 avril 1988, 132 smolts ont été marqués à l'extrémité supérieure de la caudale et ont été déversés au niveau de la station A. Au total, 57 smolts ont été retrouvés dans le piège, ce qui correspond à un pourcentage de recapture de $42.2 \%$. Le temps mis par les poissons pour retrouver le clapet a varié de $1.15 \mathrm{~h}$ à 5 jours.

Lors de la seconde opération (14 avril 1988), 87 smolts ont été marqués à l'extrémité inférieure de la caudale et ont été déversés comme précédemment au niveau de la station $A$. 46 smolts ont été recapturés dans le piège, ce qui correspond à un taux de recapture de $52.9 \%$. Les poissons ont été recapturés entre $1.30 \mathrm{~h}$ et 3 jours après leur déversement.

On ne peut malheureusement rien conclure de ces deux premières opérations car la première opération a débuté en fin de crue alors que le barrage déversait encore faiblement et un arrêt de la centrale d'une vingtaine de minutes est intervenu le jour même du lâcher ; six déclenchements consécutifs ont été enregistrés entre $19 \mathrm{~h}$ et $20 \mathrm{~h}$ le deuxième jour de la seconde expérimentation. Ces déclenchements correspondent à des arrêts imprévisibles du fonctionnement des groupes de la centrale et le débit habituellement turbiné $\left(30 \mathrm{~m}^{3} / \mathrm{s}\right)$ se déverse alors par le déversoir latéral au niveau de l'usine, puis a. horra jo. Les poissons peuvent alors franchir l'obstacle sans être crmptahilicós.

De plus, cfu-iins des smolts marqués ont pu transiter par le barrage à l'occasion de la crue ayant débuté le 18 avril, crue au cours de laquelle le débit moyen journalier a dépassé $100 \mathrm{~m}^{3} / \mathrm{s}$. 
Les opérations suivantes de marquage sont plus significatives, bien que le nombre de poissons marqués ait été plus limité, car elles n'ont été perturbées par aucun déclenchement ni déversement au barrage.

23 smolts ont été marqués à l'extrémité de la nageoire dorsale lors de la troisième opération et ont été déversés au niveau de la station A (2 mai 1988). Les smolts déversés ont mis de 2 heures à 3 jours pour parcourir le canal. 22 smolts ont été recapturés sur les 23 déversés, ce qui correspond à un taux de recapture de $96 \%$.

Lors de la quatrième opération effectuée le 9 mai 1988, 20 smolts ont été marqués au niveau de l'extrémité de la nageoire pelvienne droite et ont été déversés en amont de la station B. Ces poissons remis à l'eau le soir ont été pour 17 d'entre eux (85\%) recapturés dans la nuit (entre $4 \mathrm{~h}$ et $8 \mathrm{~h}$ ). Les deux autres ont été retrouvés dans le piège en début d'après-midi (entre 12 h et 16 h), l'opération donnant ainsi un taux de recapture de $95 \%$.

Lors de la cinquième opération, 7 smolts ont été marqués à l'extrémité de la nageoire pelvienne gauche et déversés le 10 mai à $21 \mathrm{~h}$ à la hauteur de la station $B$. 5 juvéniles déversés ont été recapturés la journée suivante (entre $4 \mathrm{~h}$ et $12 \mathrm{~h}$ ) et les deux restants le surlendemain. La totalité des effectifs déversés a donc été recapturée.

Identique à celle effectuée le 10 mai, la dernière opération a permis de marquer 8 smolts (à l'extrémité supérieure de la caudale) dont 7 ont été retrouvés dans le piège dans la journée du lendemain.

Au total, si l'on ne prend en compte que les opérations qui se sont déroulées hors période de déversement ou de déclenchement, le taux de franchissement du clapet, après marquage et déversement en amont, est voisin de $95 \%$. Tableau I : Caractéristiques et résultats des opérations de marquage en vue
d'estimer l'efficacité de l'exutoire de dévalaison.

Table $\quad$ : Characteristics and results of the tagging operations for downstream bypass structure effectiveness estimation.

\begin{tabular}{|c|c|c|c|c|c|c|c|c|c|c|c|c|c|}
\hline \multicolumn{6}{|c|}{ MARQUAGES ET DÉVERSEMENTS } & \multicolumn{7}{|c|}{ RECAPTURES } & \multirow[t]{2}{*}{ OBSERVATIONS } \\
\hline Opérations & Dates & Heure & Lieu & $\begin{array}{l}\text { Nombre de } \\
\text { smolts }\end{array}$ & $\begin{array}{l}\text { Partie de nageoire } \\
\text { sectionnee pour le } \\
\text { marquage }\end{array}$ & Jours & Heure & $\begin{array}{l}\text { Nombre de } \\
\text { smolts par } \\
\text { relevé de } \\
\text { piège }\end{array}$ & $\begin{array}{l}\text { Nombre de } \\
\text { smolts par } \\
\text { jour }\end{array}$ & $\begin{array}{l}\% \text { de } \\
\text { smolts } \\
\text { par jour }\end{array}$ & $\begin{array}{l}\text { Total } \\
\text { retour }\end{array}$ & $\begin{array}{c}\% \text { Total de } \\
\text { retour }\end{array}$ & \\
\hline $1 \cdot$ & $10 / 04 / 88$ & $10 \mathrm{H} 45$ & Station A & 132 & Caudale haut & $\begin{array}{l}10 / 04 \\
11 / 04 \\
12 / 04 \\
13: 04 \\
14: 04 \\
15 / 04 \\
\end{array}$ & $\begin{array}{l}16 \mathrm{HOO} \\
20 \mathrm{HOO} \\
24 \mathrm{HOO} \\
08 \mathrm{HOO} \\
16 \mathrm{HOO} \\
20 \mathrm{HOO} \\
24 \mathrm{HOO} \\
04 \mathrm{HOOO} \\
20 \mathrm{HOO} \\
24 \mathrm{HOO} \\
08 \mathrm{HOO} \\
16 \mathrm{HOO} \\
08 \mathrm{HOO} \\
16 \mathrm{HOO}\end{array}$ & $\begin{array}{r}2 \\
2 \\
16 \\
3 \\
1 \\
4 \\
4 \\
7 \\
1 \\
8 \\
3 \\
3 \\
1 \\
2 \\
\end{array}$ & $\begin{array}{l}20 \\
\\
12 \\
\\
16 \\
\\
6 \\
1 \\
2\end{array}$ & $\begin{array}{l}15.15 \\
9.09 \\
12.12 \\
\\
4.55 \\
0.76 \\
1.42 \\
\end{array}$ & 57 & 43.18 & $\begin{array}{l}1 \text { declenchement } \\
\text { le } 10004 \text { de } 19 \mathrm{H} 40 \\
\text { a } 19 \mathrm{H} 58\end{array}$ \\
\hline 2 & $14 / 04 / 88$ & $10 \mathrm{H} 30$ & Station A & 87 & Caudale bas & $\begin{array}{l}14 / 04 \\
15: 04 \\
16 / 04 \\
17: 04 \\
\end{array}$ & $\begin{array}{l}16 \mathrm{HOO} \\
20 \mathrm{HOO} \\
08 \mathrm{HOO} \\
16 \mathrm{HOO} \\
20 \mathrm{HOO} \\
24 \mathrm{HOOO} \\
16 \mathrm{HOO} \\
20 \mathrm{HOO} \\
24 \mathrm{HOO} \\
04 \mathrm{HOO} \\
\end{array}$ & $\begin{array}{r}2 \\
1 \\
23 \\
6 \\
4 \\
4 \\
2 \\
1 \\
1 \\
1 \\
\end{array}$ & $\begin{array}{l}3 \\
37 \\
4 \\
1 \\
\end{array}$ & $\begin{array}{r}3.45 \\
42.53 \\
4.60 \\
1.15 \\
\end{array}$ & 46 & 52.90 & $\begin{array}{l}1 \text { declenchement } \\
\text { le } 1504 \text { de } 20 \mathrm{H} 40 \\
\text { a } 20 \mathrm{H} 48 \\
6 \mathrm{declenchements} \\
\text { le } 1604 \text { de } 18 \mathrm{H} 55 \\
\text { a } 20 \mathrm{HO} 04\end{array}$ \\
\hline $3^{n}$ & $02 / 05 / 88$ & $10 \mathrm{H} 00$ & Station A & 23 & Dorsale & $\begin{array}{l}02 / 05 \\
03 / 05 \\
06 / 05 \\
07: 05\end{array}$ & $\begin{array}{l}16 \mathrm{HOO} \\
20 \mathrm{HOO} \\
04 \mathrm{HOO} \\
20 \mathrm{HOO} \\
24 \mathrm{HOO} \\
04 \mathrm{HOO} \\
20 \mathrm{HOO}\end{array}$ & $\begin{array}{l}5 \\
1 \\
2 \\
3 \\
6 \\
4 \\
1\end{array}$ & $\begin{array}{r}6 \\
11 \\
4 \\
1\end{array}$ & $\begin{array}{r}25.09 \\
47.83 \\
17.39 \\
4.35\end{array}$ & 22 & 95.65 & \\
\hline $4^{\circ}$ & $09 / 05 / 88$ & $21 \mathrm{H} 30$ & Station B & 20 & Pelvienne droite & $10 / 05$ & $\begin{array}{l}08 \mathrm{HOO} \\
16 \mathrm{HOO} \\
\end{array}$ & $\begin{array}{r}17 \\
2 \\
\end{array}$ & 19 & 95.00 & 19 & 95.00 & \\
\hline $5^{\circ}$ & $10 / 05 / 88$ & $21 \mathrm{H} 00$ & Station B & 7 & Pelvienne gauche & $\begin{array}{l}11,05 \\
12 / 05 \\
\end{array}$ & $\begin{array}{l}08 \mathrm{HOO} \\
12 \mathrm{HOO} \\
08 \mathrm{HOO} \\
\end{array}$ & $\begin{array}{l}4 \\
1 \\
2 \\
\end{array}$ & $\begin{array}{l}5 \\
2 \\
\end{array}$ & $\begin{array}{l}71.43 \\
28.57 \\
\end{array}$ & 7 & 100.00 & \\
\hline $6 \cdot$ & $12 / 05 / 88$ & $21 \mathrm{H} 00$ & Station B & 8 & Caudale haut & $13 / 05$ & $\begin{array}{l}\text { O8HOO } \\
16 \mathrm{HOO}\end{array}$ & $\begin{array}{l}6 \\
1\end{array}$ & 7 & 87.50 & 7 & 87.50 & \\
\hline
\end{tabular}




\subsection{Observations visuelles sur le comportement des smolts}

\subsubsection{Au niveau des grilles}

Lorsque le groupe situé à proximité du clapet est en fonctionnement, les smolts perçoivent assez rapidement la présence de ce dernier. Par contre, lorsque seul le groupe situé en rive gauche (à l'opposé du clapet) fonctionne, les smolts semblent éprouver plus de difficultés à trouver l'exutoire.

De façon générale, les smolts ont tendance à s'accumuler au niveau des grilles, du côté de la prise d'eau présentant les plus grandes vitesses d'approche (correspondant aux groupes en fonctionnement).

\subsubsection{Au niveau du clapet}

Le comportement des smolts au niveau du clapet semble lié aux conditions hydrauliques ambiantes.

Lorsque le clapet est fortement abaissé, en particulier pour un débit de $500 \mathrm{l} / \mathrm{s}$, et que le système fonctionne en déversoir épais, la phase exploratoire des smolts est réduite : dès que le poisson s'engage sur le radier, il se laisse emporter dans la lame d'eau. Les smolts ne semblent pas avoir de cheminement préférentiel, se répartissant pratiquement sur toute la largeur de l'exutoire avant de se laisser entraîner par l'écoulement.

Pour les débits les plus faibles, lorsque la section de contrôle se trouve au niveau de la crête déversante du clapet, le poisson semble plus hésitant à s'engager au niveau du clapet et fait preuve d'un comportement exploratoire plus long qu'aux autres débits et qui peut se prolonger pendant des heures. La répartition des poissons sur le radier s'effectue là aussi de manière uniforme sans zone de passage préférentielle.

Pour la configuration intermédiaire d'écoulement, c'est-à-dire lorsque l'écoulement s'accélère et passe en torrentiel au niveau de l'entonnement pour repasser en fluvial à l'amont du clapet, le ressaut perturbe manifestement les smolts qui se maintiennent surtout dans la partie droite de l'écoulement où le ressaut est moins marqué et la lame d'eau moins turbulente. La phase exploratoire se limite presque exclusivement à la reconnaissance de la zone de décollement située en rive droite. C'est à partir de cette zone que les poissons se laissent généralement dévaler.

Ces différents comportements ne sont pas toujours aussi tranchés car, selon la période de la migration, la durée de la phase exploratoire du poisson peut être très variable. En effet, en période de pic migratoire, les poissons descendent par bancs de plusieurs dizaines ou centaines d'individus et semblent moins exigeants sur l'importance du débit et la qualité de l'écoulement. Même à un débit de $100 \mathrm{I} / \mathrm{s}$, il est fréquent de les voir réduire considérablement leur phase d'exploration par rapport à ce qui est observé hors pic migratoire, lorsque seuls quelques individus isolés se laissent dévaler.

Il faut également noter que le tirant d'eau au-dessus du radier situé à l'amont du clapet s'est souvent trouvé très faible (de l'ordre de 20 à $40 \mathrm{~cm}$ ), ce qui oblige alors les poissons à remonter pratiquement à la surface pour pouvoir trouver puis franchir l'exutoire. Le dispositif de dévalaison aurait vraisemblablement été franchi plus rapidement avec un clapet plus étroit et un radier situé plus en profondeur au niveau de l'exutoire.

\section{DISCUSSION ET CONCLUSION}

Lors de deux années de suivi de la migration des smolts au niveau de l'usine hydroélectrique d'Halsou, l'efficacité d'un clapet de décharge aménagé en exutoire de dévalaison a été testée.

Plusieurs opérations de marquage ont été effectuées dans le but de déterminer, sur le nombre de smnlte empiuntant le canal d'amenée, la proportion transitant par l'exutoire et colle nascant par les turbines.

Hors périonn de déversement au barrage et déclenchement de l'usine, $95 \%$ des poissons marqués ont été recapturés dans le piège. Lors des deux premiers marquages, les pourcentages de recaptures ont été beaucoup plus modestes (43\% et $53 \%$ ) ; ils 
correspondent cependant à des périodes au cours desquelles ont été enregistrés plusieurs déclenchements à l'usine qui ont pu provoquer le passage de certains individus par le déversoir de crue.

Le clapet peut donc être considéré comme efficace, compte tenu des résultats précédents. Il est possible que l'inclinaison des grilles de la centrale par rapport à la direction de l'écoulement dans le canal d'amenée et l'existence d'une vitesse tangentielle aux grilles (de $0.30 \mathrm{~m} / \mathrm{s}$ à $1.0 \mathrm{~m} / \mathrm{s}$ lorsque les trois groupes sont en fonctionnement) susceptible de guider les poissons vers le clapet contribuent à l'efficacité du dispositif.

Plusieurs débits dans le clapet ont été testés. Les résultats des expérimentations des deux années tendent à montrer que l'importance du débit admis sur le clapet influence le nombre de smolts transitant par ce dernier, et ce de façon d'autant plus nette que les effectifs dévalant sont modérés.

En 1988, $17 \%$ des smolts piégés ont franchi le clapet avec un débit réglé à $100 \mathrm{l} / \mathrm{s}$, $35 \%$ avec $300 \mathrm{l} / \mathrm{s}$ et $48 \%$ avec $500 \mathrm{l} / \mathrm{s}$. La différence entre le nombre de smolts passant par le clapet à $100 \mathrm{I} / \mathrm{s}$ et $500 \mathrm{l} / \mathrm{s}$ est toujours très nette.

Outre le débit, les conditions hydrodynamiques au voisinage du clapet jouent un rôle important. L'accélération dans l'exutoire doit être progressive, en évitant toute perturbation comme les ressauts. L'écoulement doit être dans la mesure du possible "à filets parallèles".

Ces observations sur l'importance des conditions hydrodynamiques rejoignent celles effectuées par d'autres auteurs afférentes aux by-pass associés aux barrières physiques (tambours filtrants) ou comportementales (dispositifs de "louvers" ou persiennes) (BATES et VINSONHALER, 1956 ; BATES et JEWETT, 1961 ; DUCHARME, 1972 ; RUGGLES et RYAN, 1964 ; RAYNEY, 1990).

Bien qu'il puisse être considéré comme relativement efficace, le clapet d'Halsou est loin de présenter une configuration optimale : les observations visuelles montrent que le poisson reste réticent à s'engager sur le radier lorsque celui-ci n'est recouvert que par une lame d'eau de faible épaisseur. Un clapet moins large, mais plus haut et fonctionnant avec des charges plus importantes (de l'ordre de $0.50 \mathrm{~m}$ ), se révèlerait sans doute plus attractif pour le migrateur.

L'activité de dévalaison s'effectue de manière prédominante la nuit, principalement lors du pic : en 1988, plus de $70 \%$ des smolts ont dévalé la nuit. L'activité plus marquée se situe entre 22 h et $24 \mathrm{~h}$. Par contre, on observe un renversement de tendance à la fin de la migration qui peut se dérouler aussi bien le jour (78 \% en 1987, la campagne n'ayant pris en compte que la fin de la migration, et $60 \%$ la dernière semaine en 1988). Ce phénomène a déjà été remarqué par de nombreux auteurs (BAGLINIĖRE, 1976 ; THORPE et MORGAN, 1978 ; LAYZER et O'LEARY, 1978 ; NETTLES et GLOSS, 1987).

La lumière a une influence certaine sur le comportement de dévalaison nocturne des smolts. Plusieurs sources et types d'éclairage ont été testés à titre exploratoire (projecteur halogène, lampes à vapeur de mercure). Les smolts dévalent nettement mieux lorsque le clapet est maintenu dans l'obscurité que lorsqu'il est éclairé avec le projecteur halogène ou les lampes à vapeur de mercure et ce, quel que soit le débit transitant par le clapet. Cependant, les observations visuelles indiquent que les lampes à vapeur de mercure à faible puissance seraient susceptibles d'attirer le poisson, ce qui rejoint des observations effectuées par ailleurs (KAWAMURA, 1983 ; TAFT, 1988). On pourrait imaginer éclairer violemment les grilles de manière à maintenir les poissons à distance et à les guider vers l'exutoire laissé dans une semi-obscurité. L'une des causes souvent évoquée de l'entraînement du poisson dans une prise d'eau à travers des grilles, outre l'incapacité à résister aux vitesses de l'écoulement, est la perte de repères visuels dans l'obscurité (PAVLOV, 1989) : le simple fait d'éclairer les grilles peut inciter le poisson à se tenir à distance de ces dernières.

Comme pour les passes à poissons, la technique de dimensionnement des exutoires de surface, ou plus généralement des dispositifs de dévalaison, ne pourra progresser qu'en effectuant le suivi d'un certain nombre d'aménagements comme celui testé à la centrale d'Halsou. II convient cependant de prendre conscience de l'extrême lourdeur de telles opérations qui exigent la présence permanente pendant deux à trois mois de plusieurs personnes sur le site. Considérant les progrès effectués récemment dans le matériel de 
radiopistage (en particulier dans le domaine de la miniaturisation des marques), on peut désormais envisager de recourir à cette technique (NETTLES et GLOSS, 1987) comme complément, sinon alternative, aux opérations de piégeage ou de comptage par enregistrement vidéo pour l'évaluation objective de l'efficacité des dispositifs favorisant la dévalaison des juvéniles.

\section{REMERCIEMENTS}

Cette étude a été réalisée dans le cadre de conventions liant d'une part le Conseil Supérieur de la Pêche à E.D.F. (Service de la Production Hydraulique), d'autre part le CEMAGREF au Secrétariat d'État chargé de l'Environnement.

Nous tenons à remercier :

- le G.R.P.H. Pyrénées, pour avoir autorisé l'étude et tout particulièrement le personnel de la Centrale d'Halsou pour avoir contribué au bon déroulement de l'expérimentation.

- la Direction des Études et Recherches d'E.D.F. et l'INRA, station d'hydrobiologie de Saint Pée sur Nivelle, pour leur aide matérielle au cours de l'expérimentation.

- les techniciens et ingénieurs du Conseil Supérieur de la Pêche ayant participé aux différentes campagnes (MM. PUJO, CAZIN, BOUSQUET, ETCHECAHAR, FROGER, GAILLARDET, JALIBERT, MOREAU, RENARD, ROQUEFEUIL).

\section{BIBLIOGRAPHIE}

BAGLINIĖRE J.L., 1976. Activité de dévalaison des smolts sur l'Ellé. Annales d'Hydrobiologie, 7, 159-177.

BATES D.W., JEWETT S.G., 1961. Louver efficiency in deflecting downstream migrant steelhead. Trans. Am. Fish. Soc., 90 (3), p. 336-337.

BATES D.W., VINSONHALER R., 1956. Use of louvers for guiding fish. Trans. Am. Fish. Soc., $86,39-57$.

BERNARD S., LARINIER M., 1991. Suivi d'un clapet de dévalaison pour les smolts de saumon atlantique à la centrale hydroélectrique d'Halsou (64). Rapport Ghaappe 91.02, $23 \mathrm{p}$.

BOMASSI P., TRAVADE F., 1985. Projet de réimplantation du saumon dans la partie supérieure de l'Allier : expériences sur les possibilités de dévalaison des saumoneaux au barrage hydroélectrique de Poutès en 1983 et 1984 . Colloque pour la restauration des rivières à saumons. Bergerac, 28 mai-1 $1^{\text {er juin }} 1985$.

BOUSQUET B., 1981. Situation du saumon atlantique dans le bassin de l'Adour. Étude du stade smolt sur la rivière Nive. Rapport Conseil Supérieur de la Pêche, Délégation Régionale Midi-Pyrénées Aquitaine, $31 \mathrm{p}$.

BOUSQUET B., 1988. Suivi des repeuplements "saumons" réalisés sur le bassin de l'Adour en 1987. $1^{\text {re }}$ Partie : Contrôles menés au stade "tacon d'automne". Rapport Conseil Supérieur de la Pêche, Délégation Régionale Midi-Pyrénées Aquitaine, 18 p.

CONRAD V., 1986. Five downstream fish passage facilities in Nova Scotia and New Brunswick, Canada. Department of Fisheries and Oceans, Nova Scotia, July 1986, $10 \mathrm{p}$.

DAVIS J.P., FARWELL M.K., 1975. Exploits river and Indian river Atlantic salmon developments program - 1974. Newfoundlands. Res. Dev. Br. Report $n^{\circ}$ NEW/I-75-1, $55 \mathrm{p}$.

DUCHARME L.J.A., 1972. An application of louvers deflectors for guiding atlantic salmon (Salmo salar) smolts from power turbines. J. Fish. Res. Bd. Canada, 29, 1397-1404. 
EPRI, 1986. Assessment of downstream migrant fish protection technology of hydroelectric application. Stone and Webster Engineering Corporation, Boston, Massachussets, 1986

FARWELL M.K., 1972. Downstream migrant fish problems associated with hydroelectric facilities on the Exploits river. Res. Dev. Br., Newfoundlands. Prog. Rep. $n^{\circ} 86,55 p$. + app.

KAWAMURA G., 1983. Vision and behaviour of fish in the vicinity of fish lamp. Proc. Intl. Conf. Dev. Managt. Trop. Living Aquat. Resources. Serdag, Malaysia ; 2-5 Aug. 1983, p. 197-204.

LAYZER J.B., O'LEARY J.A., 1978. Out migrations of radio-tagged Atlantic salmon (Salmo salar) smolts in the Connecticut River with particular reference to the Northfield Mountain pumped storage hydroelectric project, 1976-1978. Northfield Mountain pumped storage hydroelectric anadromous fish study, part III. Report to Northeast Utilities Service Company, Berlin, Connecticut, $25 \mathrm{p}$.

NETTLES D.C., GLOSS S.P., 1987. Migrations of landlocked atlantic salmon smolts and effectiveness of a fish bypass structure at a small-scale hydroelectric facility. North American Journal of Fisheries Management, 7, 562-568.

PAVLOV D.S., 1989. Structures assisting the migrations of non-salmonid fish : USSR FAO, Fisheries Technical Paper, 308. Rome, $98 \mathrm{p}$.

RAINEY W.S., 1990. Cylindrical drum screen designs for juvenile fish protection at two large diversions. In Proceedings of the International Symposium on Fishways'90 in Gifu, Japan, October 8-10 1990, p. 143-150.

RUGGLES C.P., 1980. A review of the downstream migration of atlantic salmon. Canadian Technical Report of Fisheries and Aquatic Sciences $n^{\circ} 952$, Freshwater and Anadromous Division, Resource Branch, Department of Fisheries and Oceans, Halifax, Nova Scotia, $39 \mathrm{p}$.

RUGGLES C.P., RYAN P., 1964. An investigation of louvers as a method of guiding juvenile salmon. The Canadian Fish Culturist, vol 33, November 1964, 67 p.

RUGGLES C.P., HUTT R., 1984. Fish diversionary techniques for hydroelectric turbines intakes. Report for the Canadian Electrical Association Research and Development. Prepared by Montreal Engineering Company Limited, 5670 Spring Garden Road, Suite 801, P.O. Box 3478, South Postal Station, Halifax, Nova Scotia B3J 3J1.

SEMPLE J.R., Mc LEOD C.L., 1976. Experiments related to atlantic salmon smolts (Salmo salar) around hydroelectric turbines. Freshwater and Anadromous Division, Resource Branch. Technical Report Series n MAR/T-76-2, 23 p.

SEMPLE J.R., 1979. Downstream migration facilities and turbine mortality evaluation. Atlantic salmon smolts at Malay Falls, Nova Scotia. Fisheries and Marine Service Manuscript Report $n^{\circ} 1541$, October 1979, $15 \mathrm{p}$.

TAFT N., 1988. Evaluations of fish protection systems for use at hydroelectric plants. Hydroreview, avril 1988, 54-62.

THORPE J.E., MORGAN R.I.G., 1978. Periodicity in Atlantic Salmon (Salmo salar L.) smolt migration. Journal of Fish Biology, 12, 541-548. 